\title{
The chloride ion to the chemical oxygen demand (COD) analysis of the influence of different analysis method
}

\author{
Yan Zhao ${ }^{1}$, Hong Tang ${ }^{1, *}$, Xiaoyan Ge ${ }^{1,2}$, Yongyu $\mathrm{Li}^{1}$, Shihua Wang ${ }^{1}$ \\ ${ }^{1}$ Shanghai Urban Construction Vocational College, Shanghai, 200438, China \\ ${ }^{2}$ College of Environmental Science and Engineering, State Environmental Protection Engineering Center for Pollution Treatment and \\ Control in Textile Industry, Donghua University, 201620, Shanghai, China
}

\begin{abstract}
Research shows that chloride ion in the process of chemical oxygen demand (COD) analysis has a huge impact on the results of the analysis, How to improve the accuracy of chemical oxygen demand analysis results in the presence of chlorine ion interference is a difficult problem in current environmental monitoring work, This article introduces the chlorine ion in different analysis method to analysis the influence degree of the chemical oxygen demand (COD).
\end{abstract}

\section{Introduction}

In the process of daily monitoring and analysis, the Chemical oxygen demand of samples always with high concentration of chloride ions, but the concentration was very low. Such as, a large amount of hydrochloric acid is used in the process of rare earth industry, which leads to the high concentration of chloride ion $\left(\mathrm{Cl}^{-}\right)$in wastewater. After the chloride ion $\left(\mathrm{Cl}^{-}\right)$in the sample is oxidized by potassium dichromate, the chemical oxygen demand concentration is increased, which caused large error of the analysis results. It is also an important task of environmental monitoring to determine the COD value when the content of chloride ion is high and to produce less secondary pollution, that is, the use of highly toxic reagent $\mathrm{HgSO}_{4}$, It is also an important task of environmental monitoring. How to reduce pollution and analyze data accurately is the focus of the study.

\section{Effect of chloride ion on chemical oxygen demand analysis}

In HJ828-2017 Water quality-Determination of the. chemical oxygen demand-Dichromate method, It is pointed out that when the content of $\mathrm{Cl}^{-}$in water sample is too high, it is necessary to add mercury sulfate and other shielding agents to shield it, There are two main influencing factors:

$\mathrm{Cl}^{-}$is oxidized by oxidant, So the consumption of oxidant results in higher results, The reaction equation is:

$$
6 \mathrm{Cl}^{-}+\mathrm{Cr}_{2} \mathrm{O}_{2}-+14 \mathrm{H}^{+}=3 \mathrm{Cl}_{2}+2 \mathrm{Cr}^{3-}+7 \mathrm{H}_{2} \mathrm{O}
$$

In the reaction chloride ion is oxidized to chlorine gas, It caused chemical oxygen demand increase, The results of chemical oxygen demand was affected.
2) The reaction system add silver salt as catalyst, Chloride ions react with silver to form silver chloride precipitates that poison the catalyst, The factors affecting the results.

\section{Chloride ions to eliminate and commonly used methods}

Water quality-Determination of the. chemical oxygen demand-Dichromate method mentions that Chloride ion concentrations $1000 \mathrm{mg} / \mathrm{L}$, Dilution analysis was performed on the water samples, Therefore, dilution of water samples is one of the easiest methods, However, high dilution times of water samples with high chlorine and low COD will affect the determination accuracy. At present, Interference methods to eliminate chloride ions have emerged, There are mainly mercury sulfate shielding methods, Silver chloride precipitation, Standard curve correction method, Chlorine calibration method, Sealed digestion method, KI-KMnO4oxidation method, In this paper, combined with laboratory analysis data between different methods of application were analyzed.

\subsection{Mercury sulfate shielding}

The national standard mentions the use of $\mathrm{HgSO}_{4}$ to shield chloride ions, $\mathrm{HgSO}_{4}$ is used as chloride ion masking agent, This method is effective for low mass concentration of chloride ion, The results were still higher when the chloride concentration was very high, And the error increases with the increase of chloride ion concentration, $\mathrm{HgSO}_{4}$ itself is highly toxic, The mercury salts in the waste solution are difficult to dispose, And it will cause secondary pollution to the environment, Therefore, it is not feasible to add sufficient $\mathrm{HgSO}_{4}$ reagent. 


\subsection{Silver salt precipitation}

Silver salt precipitation is the process of adding silver nitrate to water samples, So precipitate the chloride ions into silver chloride. The method is suitable for the sample chlorine ion greater than $10000 \mathrm{mg} / \mathrm{L}$, This method usually takes two forms: One is the addition of $\mathrm{AgNO} 3$ during preprocessing, The supernatant was taken for COD determination. This method requires $\mathrm{AgNO}_{3}$ to be added appropriately, Allow chloride ions to precipitate completely and not overaccumulate, Yufeng Liu et al [1] neutralized the chloride ion with silver nitrate based on the standard method, The acidity of the reaction system was also increased, The pollution of mercury salt was avoided, The experimental results was satisfactory, The COD standard samples were prepared by using $\mathrm{NaCl}$ solutions of different concentrations, After removing the chloride ion by the above method, The experimental results show that The COD value measured after precipitation is lower than the standard value, It can be seen that during $\mathrm{AgCl}$ precipitation, organic matter in water samples will be partly removed by co-precipitation and flocculation, That maked the measurement result lower. In addition, precious silver salts are used in the sedimentation process, Raise the cost of measurement, Therefore, it is necessary to recycle and reuse silver. Therefore, this method is not feasible in the case of muddy water samples.

\subsection{Standard curve correction method}

1) Method and principle

Different concentration of chloride ion aqueous solution with sodium chloride, With the national standard method for determination of COD concentration, Plot the measured results as a standard curve, The results show that chlorine ion in the certain concentration range and has a good linear relationship between COD, However, the higher the chloride content, the less obvious the titration endpoint was, The concrete results in the following table.

Table1. The relationship between $\mathrm{CL}^{-}$and cod

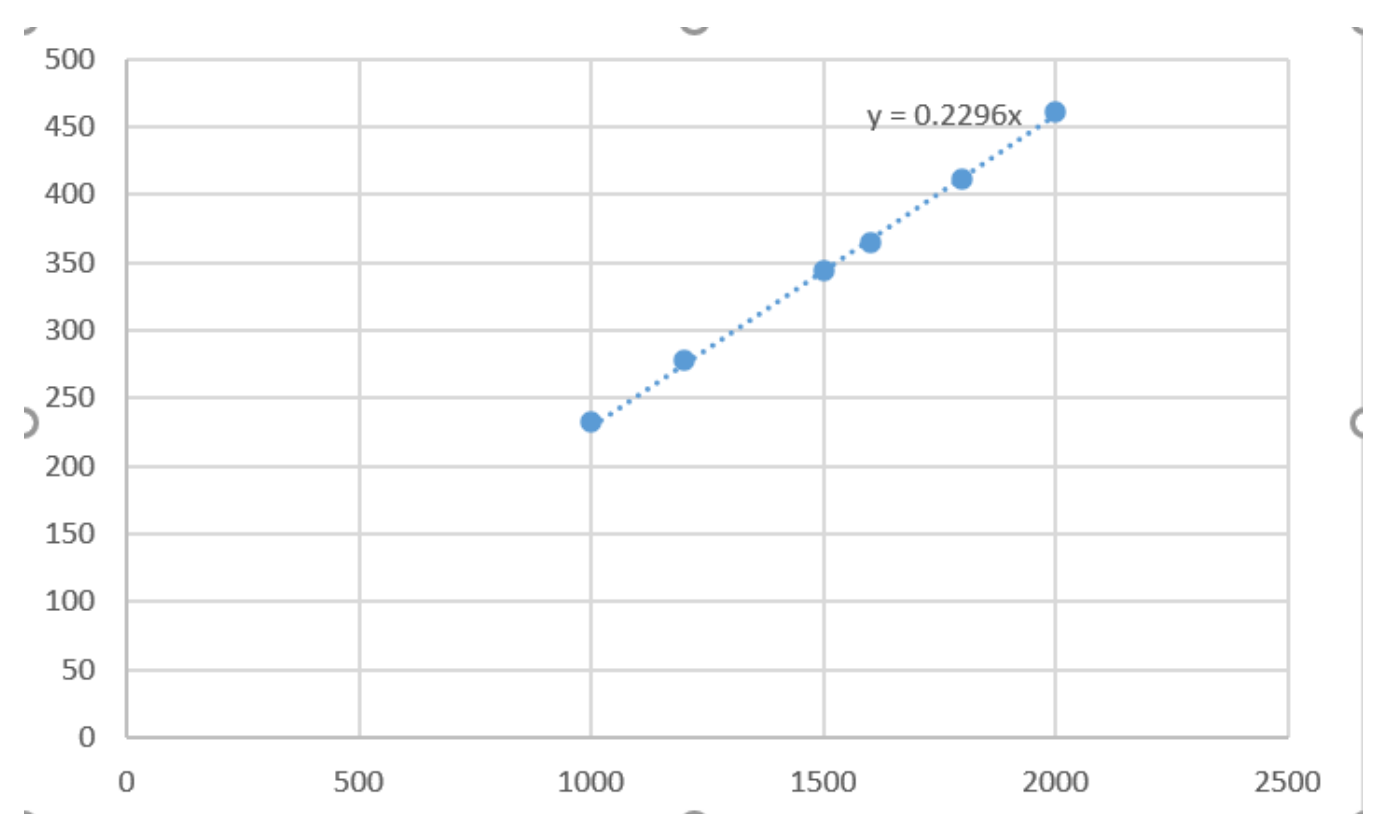

Steps of standard curve correction method Take two samples of the same water samples, one of which did not mask $\mathrm{Cl}^{-}$to determine the COD, which was recorded as the total COD, and the other was used to determine the chloride ion content. Find out the corresponding COD value on the standard curve and record it as $\mathrm{COD}_{\mathrm{cl}-\text {, }}$ then the difference between the total COD and $\mathrm{COD}_{\mathrm{cl}}$-is the real COD value of the sample.

The calibration method of standard curve does not use mercury salt and silver salt, which was environmentally friendly and economical. Junxia Wang [2] and others have proved through experiments that this method of complete oxidation is equivalent to the oxygen consumption when the theoretical $\mathrm{Cl}^{-}$is completely oxidized, and the $\mathrm{Cl}^{-}$ oxidation rate is more than $99 \%$, and the measured value of cod has good consistency with the actual value. However, due to the different methods and operating conditions used in each laboratory, the oxidation degree of $\mathrm{Cl}^{-}$is different, so the standard curves drawn by different people were not the same. Moreover, the determination of $\mathrm{Cl}^{-}$concentration in each sample was complicated. So the application of this method is limited.

\section{4 chlorine calibration method}

In chlorine calibration method, a reflux absorption device was used in the digestion of chlorine gas. The $\mathrm{Cl}_{2}$ generated by oxidation during the digestion of chemical oxygen demand is exported to $\mathrm{NaOH}$ solution for absorption, and the standard solution of $\mathrm{Na}_{2} \mathrm{~S}_{2} \mathrm{O}_{3}$ is used for titration. The consumption of $\mathrm{Na}_{2} \mathrm{~S}_{2} \mathrm{O}_{3}$ was converted into the consumption of oxygen, which was the correction value of $\mathrm{Cl}^{-}$. The actual COD value of wastewater is the difference between the apparent COD value and the corrected value of $\mathrm{Cl}^{-}$[4]. The method was suitable for the determination of high chlorine wastewater with chlorine ion content less than $20000 \mathrm{mg} / \mathrm{L}$ and COD greater than $30 \mathrm{mg} / \mathrm{L}$. This method requires very strict experimental 
conditions.

This method is the most widely used method in the determination of high chlorine. The following points should be paid attention to in the analysis:

Flow control: in the negative process, the flow rate of nitrogen blowing should be strictly controlled. The flow rate is related to the absorption efficiency of $\mathrm{NaOH}$. In the digestion process, the pipeline connection should be strictly checked to avoid air leakage.

According to the standard, the flow rate of nitrogen is $5 \sim 10 \mathrm{ml} / \mathrm{min}$. the absorption efficiency is the highest when the flow rate is about $10 \mathrm{ml} / \mathrm{min}$.

The amount of $\mathrm{HgSO}_{4}$ added should be strictly controlled. Too little $\mathrm{HgSO}_{4}$ will lead to the reaction of $\mathrm{Cl}^{-}$ and $\mathrm{AgNO} 3$ to produce $\mathrm{AgCl}$ precipitation, which will affect the determination. Excessive addition will cause waste of reagents and secondary pollution.

When using $\mathrm{Na}_{2} \mathrm{~S}_{2} \mathrm{O}_{3}$ standard solution, the reaction is fast, and the titration speed is slow. After shaking it, titrate the next drop.

When the $\mathrm{pH}$ value of the absorption solution was controlled, that is, the amount of sulfuric acid was controlled. When the $\mathrm{pH}$ value of the absorption solution was $2 \sim 4$, the error of the determination result is the least, so the amount of sulfuric acid added was particularly important.

Finally, the limitation of this method was that it was not suitable for the analysis of high concentration chloride ion and low concentration sample, In the follow-up work, the improved equipment and method will be verified.

\section{$3.5 \mathrm{KI}^{-\mathrm{KMnO}_{4}}$ oxidation method}

In the alkaline condition, $\mathrm{KMnO}_{4}$ is used to oxidize the substances in the wastewater, the remaining $\mathrm{KMnO}_{4}$ is reduced by $\mathrm{Ki}$, and then titrated with $\mathrm{Na}_{2} \mathrm{~S}_{2} \mathrm{O}_{3}$ standard solution, and the consumption of $\mathrm{Na}_{2} \mathrm{~S}_{2} \mathrm{O}_{3}$ is converted into the consumption of oxygen, so as to obtain a COD value. However, the measured value of this method is different from that of $\mathrm{K}_{2} \mathrm{Cr}_{2} \mathrm{O}_{7}$ oxidation method. There is a ratio $\mathrm{K}$ between them. Therefore, the $\mathrm{COD}$ value measured by $\mathrm{KI}^{-\mathrm{KMnO}_{4}}$ oxidation method can be converted only by knowing the ratio $\mathrm{K}$. This method is suitable for the wastewater with the content of $\mathrm{Cl}^{-}$ranging from tens of thousands to hundreds of thousands of $\mathrm{mg} / \mathrm{L}$, but the $\mathrm{K}$ value needs to be determined, which brings great inconvenience to the monitoring work. In addition, the use of highly toxic reagent sodium azide in the determination process will cause great pollution to the environment, and at the same time, there is a potential safety hazard to the analysts.

\section{Conclusion}

In conclusion, the main methods to eliminate the interference of $\mathrm{Cl}^{-}$on COD determination are reagent shielding and determination correction. The principle of our method is that it is simple, quick and effective and does not produce secondary pollution, In the application of the existing analysis methods, we can see that each method has a certain scope of application and limitations. In the future research process, I think we can combine the existing methods to improve or use the cross infiltration between the methods to improve the COD analysis of high concentration chloride ion.

\section{Reference}

1. Yufeng Liu, Dongfang Qu, Li Wang. Study on Rapid Determination of $\mathrm{COD}_{\mathrm{Cr}}$ in high chloride ion wastewater [J]. Groundwater. 2008.30 (5): 129-130

2. Junxia Wang, Wencai Wang, Junrong Wang. Analysis technology of high chloride ion and low concentration cod water sample [J]. China environmental monitoring. 2006.22 (2): 4-6

3. Editorial board of the State Environmental Protection Administration of the people's Republic of China. Water and wastewater monitoring and analysis methods (Fourth Edition supplement) [M]. Beijing: China Environmental Science Press. 2006:220-223

4. Determination of chemical oxygen demand in high chlorine wastewater by chlorine correction method HJ / t70-2001

5. Water quality-Determination of the. chemical oxygen demand-Dichromate method, HJ828-2017 